\title{
Dichloroacetate enhances the antitumor efficacy of chemotherapeutic agents via inhibiting autophagy in non-small-cell lung cancer
}

This article was published in the following Dove Press journal:

Cancer Management and Research

\author{
Xiao Lu ${ }^{1, *}$ \\ Dong Zhoul,* \\ Bing Hou' \\ Quan-Xing Liu' \\ Qian Chen ${ }^{2}$ \\ Xu-Feng Deng ${ }^{1,3}$ \\ Zu-Bin Yu' \\ Ji-Gang Dai' \\ Hong Zheng' \\ 'Department of Thoracic Surgery, \\ Xinqiao Hospital, Third Military \\ Medical University, Chongqing, \\ People's Republic of China; 'Institute \\ of Pathology and Southwest \\ Cancer Center, Southwest Hospital, \\ Third Military Medical University, \\ Chongqing, People's Republic of \\ China; ${ }^{3}$ Department of Cardiothoracic \\ Surgery, First People's Hospital of \\ Zunyi, Guizhou, People's Republic of \\ China
}

*These authors contributed equally to this work

Correspondence: Hong Zheng;

Ji-Gang Dai

Department of Thoracic Surgery,

Xinqiao Hospital, Third Military Medical

University, No. 183, Xinqiao Street,

Shapingba District, Chongqing 400037,

People's Republic of China

Tel +86 2368774724

Fax +862368774724

Email ziecoe@I63.com;691057831@

qq.com
Background: Chemotherapy is still the primary adjuvant strategy of cancer therapy; however, the emergence of multi-drug resistance has been a cause for concern. Autophagy has been demonstrated to have a protective role against chemotherapeutic drugs in cancer cells, and autophagy inhibition is generally considered to be a promising therapeutic strategy. However, the paucity of effective and specific autophagy inhibitors limits its application.

Purpose: The objective of this study was to explore the effect of DCA, small molecular antitumor agent, on the autophagy regulation and chemosensitization in NSCLC cells.

Methods: We investigated the autophagy regulation of dichloroacetate (DCA) by laser confocal microscopy and western blotting in A549 and H1975 cell lines. The MTT assay and flow cytometry was performed for explore the chemosensitization effectiveness of DCA. The results were verified with subcutaneous tumor model in nude mice and the immunohistochemistry was applied for assessing the level of cell apoptosis and autophagy in vivo post treatment.

Results: We found that DCA, which exhibited antitumor properties in various carcinoma models, induced apoptosis of non-small cell lung cancer cells (NSCLC) by inhibiting cancer cell autophagy. Furthermore, Perifosine, an AKT inhibitor, can greatly weaken the capacity of inducing apoptosis by DCA. The results indicate that the AKT-mTOR pathway, a main negative regulator of autophagy, is involved in the DCA-induced inhibition of autophagy. Then, we detected the effectiveness of autophagy inhibition by DCA. When used in co-treatment with the chemotherapeutic drug paclitaxel (PTX), DCA markedly decreased cell autophagy, enhanced apoptosis and inhibited proliferation in A549 and H1975 cells. The results of the xenograft experiment demonstrate that co-treatment of PTX and DCA can significantly decrease cell proliferation in vivo and prolong the survival of mice.

Conclusion: Our results suggest that DCA can inhibit cell autophagy induced by chemotherapeutics, providing a new avenue for cancer chemotherapy sensitization.

Keywords: DCA, autophagy, multi-drug resistance, non-small-cell lung cancer, paclitaxel, xenograft nude mice, chemosensitization

\section{Introduction}

Non-small-cell lung cancer (NSCLC) is one of the leading causes of cancer mortality worldwide. It is the most commonly occurring cancer in men and women, with an incidence greater than the combined incidence of breast, cervical, and colorectal cancers. ${ }^{1,2}$ Although chemotherapy is still the most important means of adjuvant therapy for inoperable cancer patients and patients undergoing surgery, the clinical benefits of platinum- and paclitaxel-based postoperative chemotherapies are modest, especially in advanced NSCLC. At the same time, the adverse drug reactions have become 
more severe, and drug resistance has also been emerging. ${ }^{3}$ Therefore, the need for novel strategies to replace/supplement traditional chemotherapy is urgent.

Growing evidence in recent years has shown that tumor cells preferentially produce energy for cell growth and division through glycolytic process and lactic fermentation. The rates of anaerobic metabolism and glycolysis in rapidly growing malignant tumor cells are significantly higher than those in normal cells. This reprogramming of energy metabolism is known as the Warburg effect, and it can be exploited as a therapeutic target to inhibit tumor growth. Among numerous drugs that target metabolism, dichloroacetate (DCA) has shown excellent potential because of its positive contribution to cancer treatment. ${ }^{4,5}$

Another mechanism that is completely altered in cancer cells is autophagy, a homeostatic cellular degradation system that is responsible for degrading damaged or unnecessary cellular organelles or proteins. ${ }^{6}$ During autophagy, cellular cargo destined for degradation is enclosed in an autophagosome, a double-membraned vesicle. The loaded autophagosome fuses smoothly with a lysosome to form an autolysosome, where the delivered cellular material is degraded by various lysosomal hydrolytic enzymes. The autophagy process has been researched extensively. It is becoming increasingly clear that altered autophagy activity is associated with tumor formation and progression..$^{7-9}$ Because autophagy plays a protective role in cancer cells against chemotherapeutic drugs, autophagy suppression during chemotherapy has been considered a novel therapeutic strategy. ${ }^{10-12}$ At present, only chloroquine (CQ) is being used in clinical settings as an efficacious autophagy inhibitor. Although the efficacy and feasibility of chloroquine in cancer therapy have been demonstrated, the unwanted side effects might be the problem for clinical treatment. Discovering and using additional autophagy inhibitors in cancer therapy would be of great clinical significance. ${ }^{13-15}$

DCA is a mitochondria-targeting agent that acts as a metabolic switch, reversing the abnormal cancer cell metabolism from anaerobic glycolysis to aerobic glucose oxidation by reducing the activity of mitochondrial PDK1 and enhancing the viability of PDH. Thus, DCA increases mitochondrial reactive oxygen species, thereby inducing apoptosis in malignant tumor cells without affecting normal cells. ${ }^{16,17}$ However, the regulatory action of DCA for autophagy in lung cancer is still unclear. In this study, we demonstrated that DCA inhibited cell proliferation and enhanced tumor cell apoptosis through the downregulation of autophagy, thus increasing the efficiency of cell death when used in cotreatment with chemotherapeutic agents.

\section{Materials and methods \\ Cell culture and reagents}

Human lung adenocarcinoma A549 cells were cultured in Dulbecco's Modified Eagle Medium with 10\% fetal bovine serum, and H1975 cells were cultured in Roswell Park Memorial Institute-1640 medium with 10\% fetal bovine serum in a humidified incubator with $95 \%$ air and $5 \% \mathrm{CO}_{2}$ at $37^{\circ} \mathrm{C}$. The A549 (TCHu150) and $\mathrm{H} 1975$ (TCHu193) cell lines were purchased from the Library of Typical Culture of the Chinese Academy of Sciences (Shanghai, People's Republic of China). After the cells grew along the wall of the culture dish, $0.25 \%$ trypsin (HyClone, Buckinghamshire, UK) was used for detaching and subculturing. The reagents used in this study were DCA (Sigma-Aldrich, St. Louis, MO, USA), Paclitaxel (PTX; Sigma-Aldrich), Cisplatin (cis-diamminedichloroplatinum [CDDP]; Sigma-Aldrich), adenovirus (GFP-RFP-LC3; Hanbio, Shanghai, People's Republic of China), TUNEL (terminal deoxynucleotidyl transferase dUTP nick end labeling; Promega, Fitchburg, WI, USA), APC-Annexin V, propidium iodide (BD Pharmingen, Franklin Lakes, NJ, USA), and MTT kit (Sangon Biotech, Shanghai, People's Republic of China). The antibodies were LC3-I/II, p62, PARP (all Abcam, Cambridge, UK), $\beta$-actin (Sigma-Aldrich), mTOR/p-mTOR (Sigma-Aldrich), and Ki-67 (Biovisualab, Shanghai, People's Republic of China).

\section{Immunofluorescence microscopy}

Cells $\left(1 \times 10^{5}\right)$ were inoculated in a 24 -well plate and incubated overnight. Prior to the experiment, the cells were infected with adenovirus containing tandem GFP-RFP-LC3 structures. At $24 \mathrm{~h}$ postinfection, the media were changed, and the cells were treated with $25 \mathrm{mM}$ DCA or Hank's balanced salt solution (HBSS; Sigma Aldrich) for $24 \mathrm{~h}$. For immunostaining, the cells were fixed in $4 \%$ paraformaldehyde and washed with PBS. The cells were incubated with DAPI (4',6-diamidino2-phenylindole; Beyotime Biotechnology, Shanghai, People's Republic of China) for $5 \mathrm{~min}$ and washed thrice with PBS. The coverslips were mounted on slides with mounting medium. The images were captured using an LSM 780 Meta confocal microscope (Carl Zeiss MicroImaging $\mathrm{GmbH}$, Jena, Germany) and processed using the software provided by the manufacturer.

\section{Apoptosis assay}

Cells $\left(1 \times 10^{5}\right)$ were inoculated in a 24 -well plate and incubated overnight. Prior to the experiments, the cells were treated with different concentrations of $25 \mathrm{mM}$ DCA, chemotherapeutic agent plus DCA or other reagents for $24 \mathrm{~h}$. The cells were stained with APC-Annexin V and propidium iodide 
to measure the rate of apoptosis by flow cytometry. Each experiment was repeated three times.

\section{Western blot}

Cells were lysed on ice by treatment with radioimmunoprecipitation assay (RIPA) lysis buffer (Sangon Biotech) with Halt ${ }^{\mathrm{TM}}$ Protease and Phosphatase Inhibitor Cocktail (Thermo Fisher Scientific, Waltham, MA, USA) for $15 \mathrm{~min}$. The supernatants were collected after centrifugation. The protein concentrations were measured using the bicinchoninic acid (BCA) protein assay kit (Beyotime Biotechnology). Protein samples were electrophoresed on $10 \%$ sodium dodecyl sulfate-polyacrylamide gels and transferred onto an Immobilon PVDF membrane. The membranes were probed overnight at $4^{\circ} \mathrm{C}$ with the indicated primary antibody and then incubated with a horseradish peroxidase-conjugated secondary antibody. Bands were visualized using chemiluminescence detection buffer (Takara, Shiga, Japan), and the band intensity was quantified by software provided by the manufacturer.

\section{Cell viability assay}

Cells were cultured in 96-well plates $(1,000$ cells in 100 $\mu \mathrm{L}$ culture medium/well). After drug treatment, $10 \mu \mathrm{L}$ MTT $(0.5 \mathrm{mg} / \mathrm{mL})$ was added and incubated for $4 \mathrm{~h}$. The medium was then discarded, and the formazan crystals were solubilized by the addition of DMSO (dimethyl sulfoxide; Sigma-Aldrich). The absorbance at $570 \mathrm{~nm}$ was measured. The cell viabilities were normalized to that of the control group.

\section{In vivo tumor xenograft studies}

For this assay, $5 \times 10^{6}$ cells were subcutaneously injected into nude mice (BALB/c, Specific pathogen free grade, 4-5 weeks of age, purchased from the Model Animal Research Center of Nanjing University, Nanjing, People's Republic of China). When the tumor size reached $100 \mathrm{~mm}^{3}$ (15-20 days), mice were randomly divided into 4 groups (PBS, PTX, DCA, and PTX plus DCA). The dosage of PTX was $20 \mathrm{mg} / \mathrm{kg} / \mathrm{d}$, and the dosage of DCA was $100 \mathrm{mg} / \mathrm{kg} / \mathrm{d}$. The length and width of the tumors were measured every two days across their two perpendicular diameters (calculated volume $=$ shortest diameter ${ }^{2} \times$ longest diameter/2). The number and dates of deaths of the mice were recorded to calculate the survival rate. All manipulations involving live mice were approved by the Animal Care and Use Committee of Xinqiao Hospital and followed the Chinese Guideline of Welfare and Ethics for Laboratory Animals, and all efforts were made to minimize suffering.

\section{Histological examination}

All tumors were resected after $24 \mathrm{~d}$ for histopathological examination. Sections (4- to 5- $\mu \mathrm{m}$ thick) of the tumor tissues were fixed with $10 \%$ formalin and then detected with Ki-67, LC3B antibody, and TUNEL assay. The sections were washed thrice and treated with diaminobenzidine for color development. Histological examinations were performed, and photographs were taken using a NanoZoomer 2.0-RS optical microscope (Hamamatsu Photonics, Hamamatsu City, Japan).

\section{Statistical analysis}

The data are presented as the mean \pm standard deviation. One-way analysis of variance and independent sample $t$-test were used to analyze the variance. Survival curves were obtained by the Kaplan-Meier method, and comparisons were made by the log-rank test. All statistical analyses were conducted using SPSS 19.0 statistical software (SPSS; IBM Corporation, Armonk, NY, USA). $P$-values $<0.05$ or $<0.01$ were considered statistically significant.

\section{Results}

\section{DCA inhibited autophagy in lung adenocarcinoma cells}

Cytosolic LC3 and SQSTM (p62) are highly conserved proteins that are considered to play essential roles during key stages of autophagy. To investigate the role of DCA in autophagy regulation, we examined the cellular localization of LC3 with a confocal laser-scanning microscope. A549 and H1975 cells were infected with adenovirus encoding a tandem GFP-RFP-LC3 construct to detect the change in autophagy flux. A difference in the $\mathrm{pH}$ sensitivity can cause the varying degrees of GFP-LC3 and RFP-LC3 protein accumulation in neutral autophagosomes and acidic autolysosomes. After the treatment with HBSS, the number of GFP or RFP-LC3 dots was significantly increased, demonstrating that HBSSinduced starvation significantly promoted the autophagy flux in A549 and H1975 cells. However, the DCA treatment successfully inhibited the expression of LC3 proteins relative to the control or HBSS treatment both in A549 cells and H1975 cells (Figure 1A and D, $p<0.01$ ).

p62 protein accumulates when autophagy is inhibited and decreases when autophagy is induced. Western blotting analysis was performed to examine the protein levels of $\mathrm{p} 62$ and LC3-II following DCA treatment. We observed a significant decrease in the levels of LC3 and increased p62 following DCA treatment in a concentration-dependent manner both in A549 and H1975 cells (Figure 1B and E, $p<0.01$ ). Autophagy was enhanced during incubation with HBSS, which was reversed when cotreated 
A
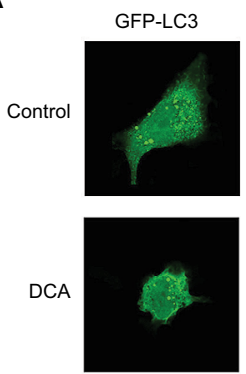

HBSS
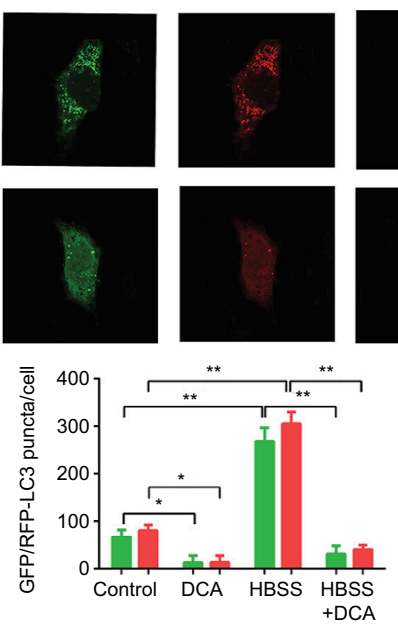

D
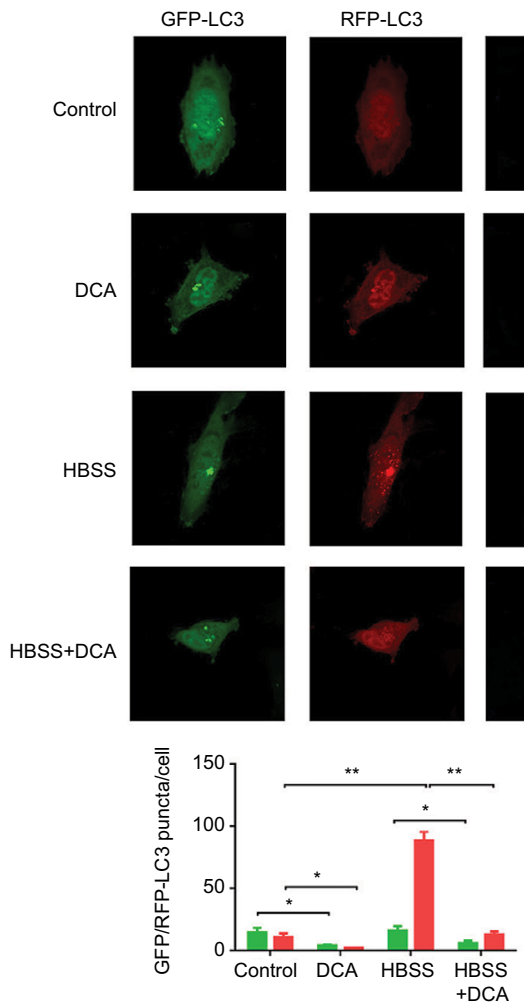

B
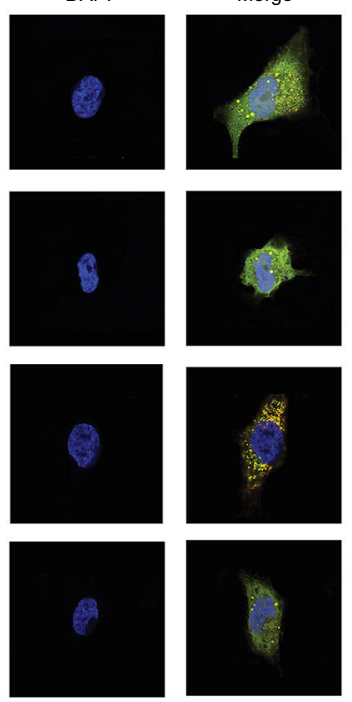

GFP-LC3

RFP-LC3

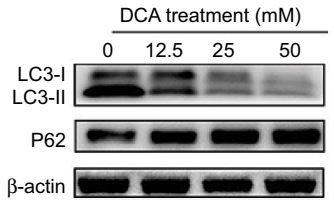

LC3-II/ $\beta$-actin
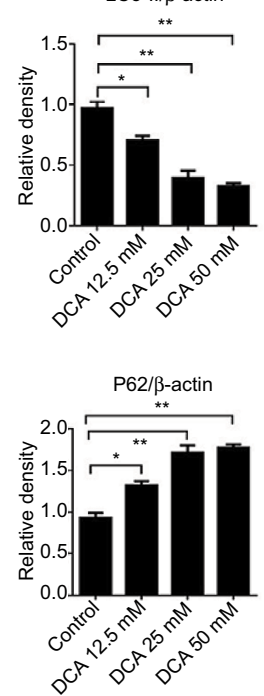

E
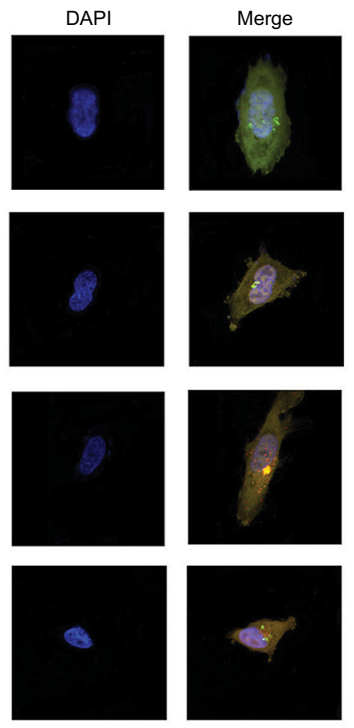

GFP-LC3
RFP-LC3

$$
\text { RFP-LC3 }
$$
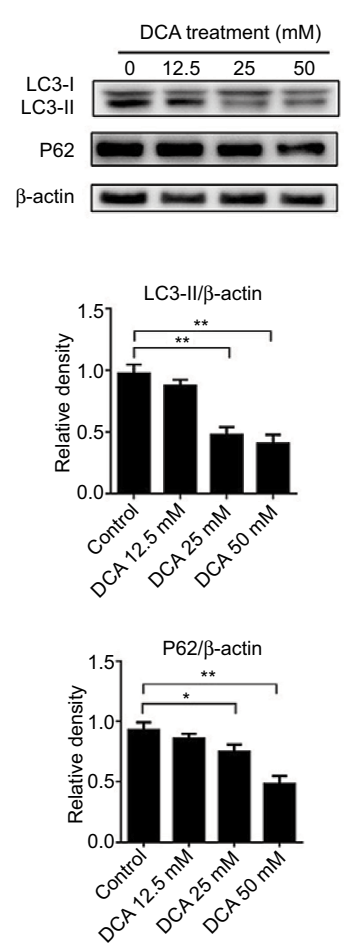

C

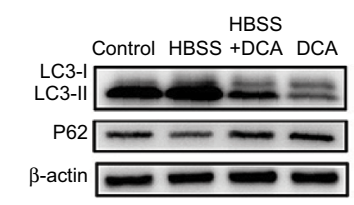

LC3-II/ $\beta$-actin

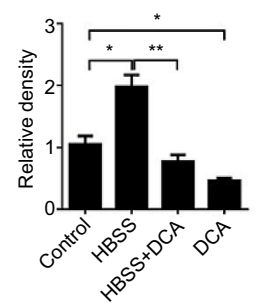

P62/ß-actin

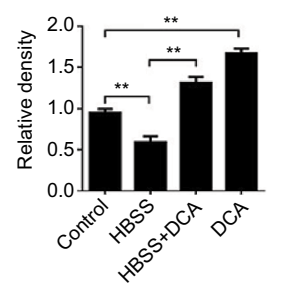

$\mathbf{F}$

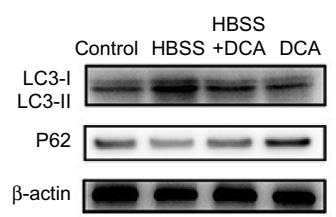

LC3-II/ $/$-actin

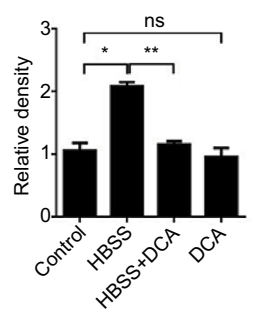

P62/ $\beta$-actin

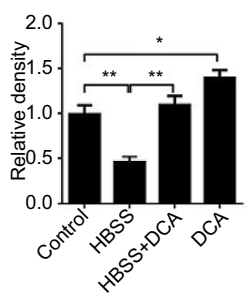

Figure I DCA inhibited autophagy in human lung cancer cells.

Notes: (A) A549 cells and (D) HI975 cells were infected with GFP-RFP-LC3 adenovirus and incubated $24 \mathrm{~h}$ before DCA treatment. After treatment with 25 mM DCA for $24 \mathrm{~h}$, the cells were then stained with DAPI to determine the nucleus. The expression of LC3 was analyzed by fluorescence microscopy. The puncta of GFP/RFP-LC3 per cell was analyzed by ImageJ. The data are presented as the mean \pm SD, ${ }^{*} p<0.05$, ${ }^{* *} p<0.01$. (B) A549 cells and (E) HI 975 cells were treated with I2.5 mM, $25 \mathrm{mM}$, or $50 \mathrm{mM}$ DCA for $24 \mathrm{~h}$, and (C and F) the cells were also treated with HBSS, DCA, or coincubation for $24 \mathrm{~h}$. Then, the level of LC3B-Il and p62 was analyzed by Western blot. The relative density of autophagy-related proteins was statistically estimated and compared, and the data are presented as the mean $\pm S D(* p<0.05$, $* * p<0.01)$.

Abbreviations: DAPI, 4',6-diamidino-2-phenylindole; DCA, dichloroacetate; HBSS, Hank's balanced salt solution; ns, not significant. 
A
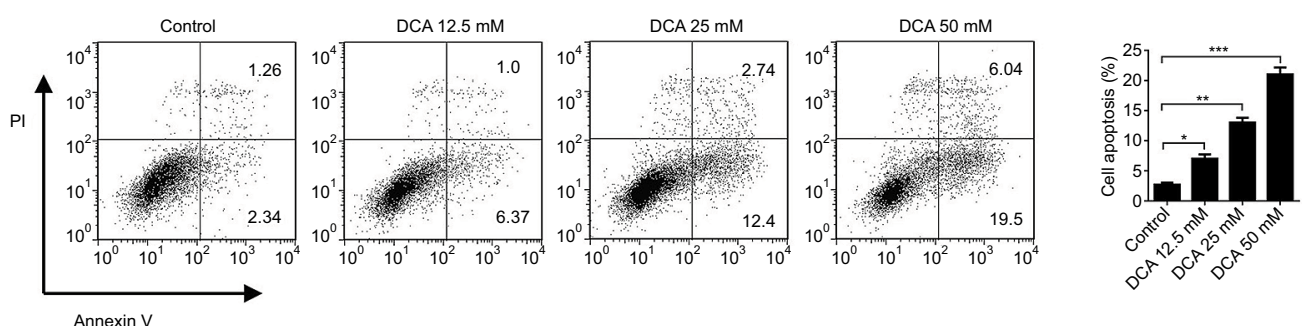

B

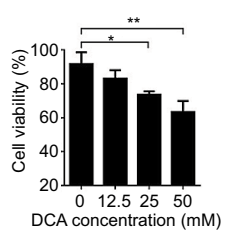

C

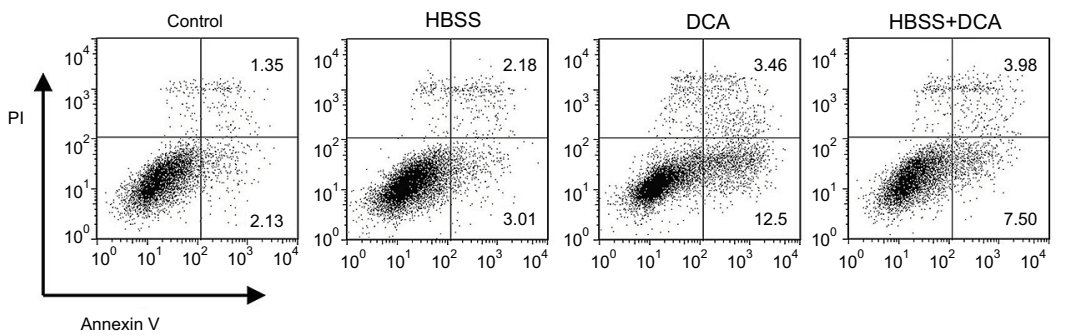

E
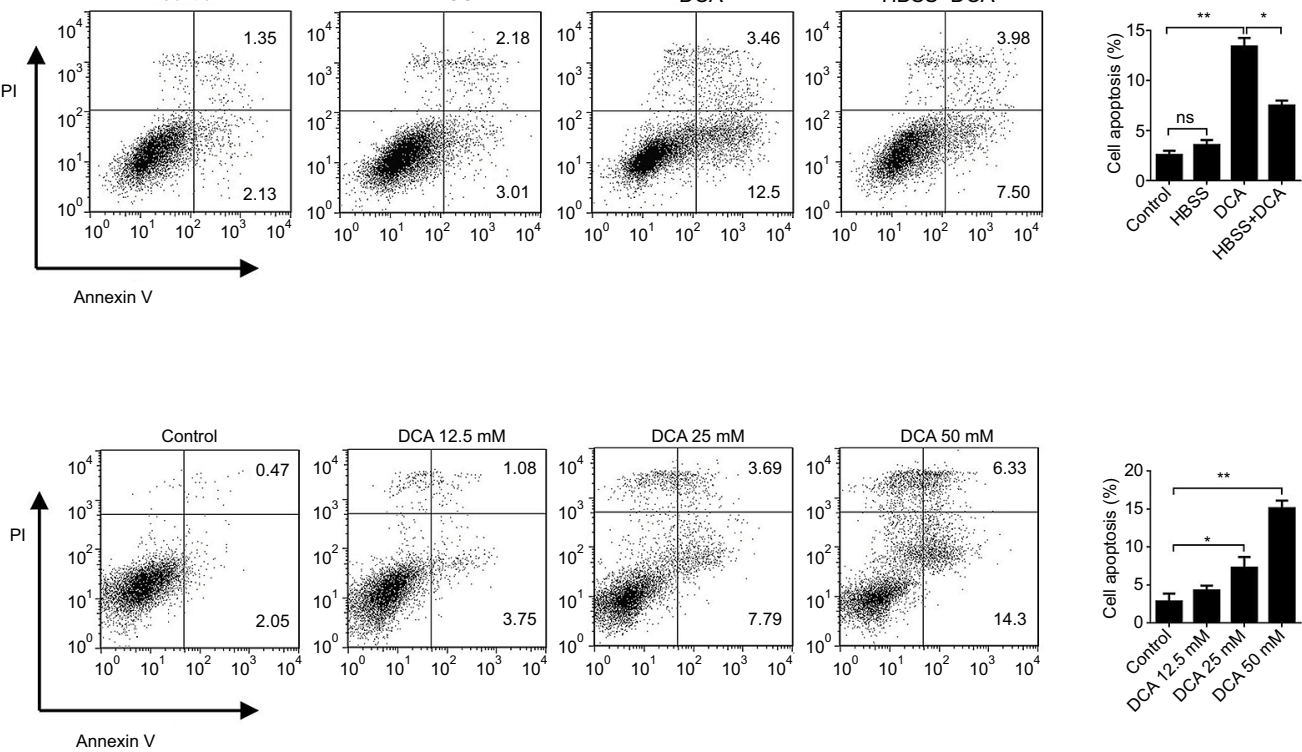

D

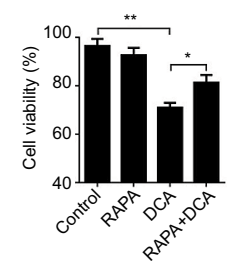

F

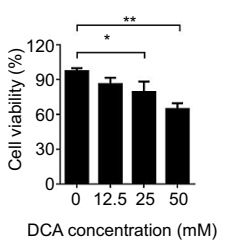

H

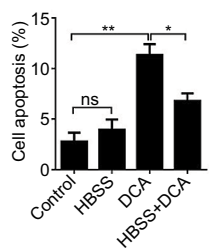

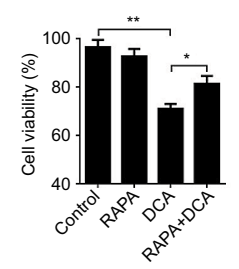

Figure 2 DCA treatment induced potent cell death through autophagy inhibition.

Notes: (A) A549 cells and (C) HI975 cells were treated with $12.5 \mathrm{mM}, 25 \mathrm{mM}$, or $50 \mathrm{mM}$ DCA for 24 h. (E and G) The cells were treated with HBSS, DCA, or coincubation for $24 \mathrm{~h}$. Then, the cell apoptosis was analyzed by Annexin V-FITC/PI staining and flow cytometry. The apoptosis data are presented as the mean \pm SD from three independent experiments $\left({ }^{*} p<0.05\right.$, $\left.{ }^{* *} p<0.0 \mathrm{I}\right)$. (B) A549 cells and (D) HI 975 cells were treated with different concentrations of DCA (F and $\left.\mathbf{H}\right)$ or treated with HBSS, DCA, or coincubation with HBSS plus DCA. Then, the cell viability was determined by MTT assay. The data are presented as the mean \pm SD from three independent experiments $\left(* p<0.05,{ }^{* *} p<0.01\right)$.

Abbreviations: DCA, dichloroacetate; FITC/PI, fluorescein isothiocyanate/propidium iodide; HBSS, Hank's balanced salt solution.

with DCA (Figure $1 \mathrm{C}$ and F, $p<0.01$ ). Taken together, these results indicate that the autophagic flux in A549 and H1975 cells was significantly inhibited by the DCA treatment.

\section{The effect of autophagy promotion on cell death induced by DCA}

Systematic research on the anticancer effects of DCA was performed. First, the anticancerous ability of DCA was examined in vitro. Both cells were incubated for $24 \mathrm{~h}$ in the presence of different concentrations of DCA, and cell apoptosis and viability were assessed. We observed that the anticancer ability of DCA, as reflected in the cell viability inhibition and apoptosis rates, increased in a concentration-dependent manner in A549 cells and H1975 cells (Figure 2A and B, E and F, $p<0.01$ ).

DCA activated the metabolic switch that reversed the abnormal metabolism in cancer cells from anaerobic glycolysis 
to glucose oxidation, which resulted in mitochondrial dysfunction and cell death. Our preliminary experiments showed that autophagy was significantly inhibited by DCA treatment. To determine whether the DCA-mediated autophagy inhibition was involved in apoptosis, A549 and H1975 cells were treated with DCA after HBSS for $24 \mathrm{~h}$. Cell apoptosis induced by DCA was inhibited when the cells were coincubated with HBSS compared with the DCA treatment alone. Except for altering the abnormal metabolism, this result confirms that DCA can also activate cell death by inhibiting autophagy in cancer cells. The cell viability results are consistent with this conclusion (Figure 2C and D, G and H, $p<0.05$ ).

\section{Inhibition of the AKT pathway decreased DCA-induced apoptosis}

The ATK-mTOR pathway has been considered to play a critical role in autophagy regulation in previous studies. ${ }^{18-21}$ Perifosine, ${ }^{22}$ a chemical reagent preventing AKT phosphory-
A
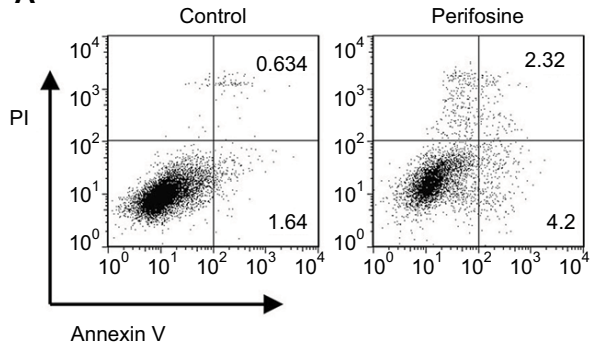

Annexin

C

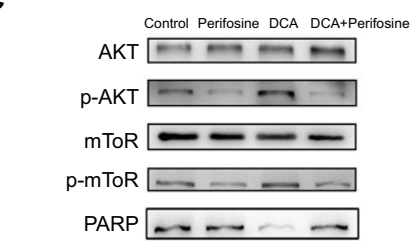

Pro-caspase $3 \longrightarrow \cdots$

$\beta$-actin

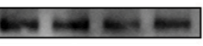

D

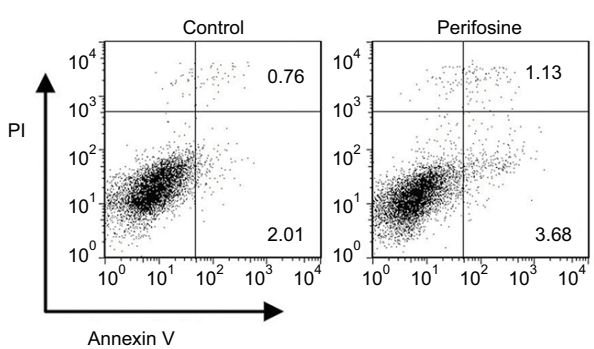

DCA

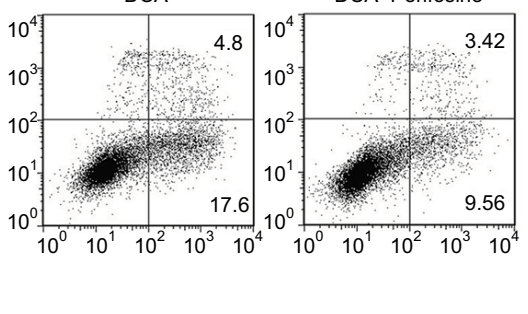

B
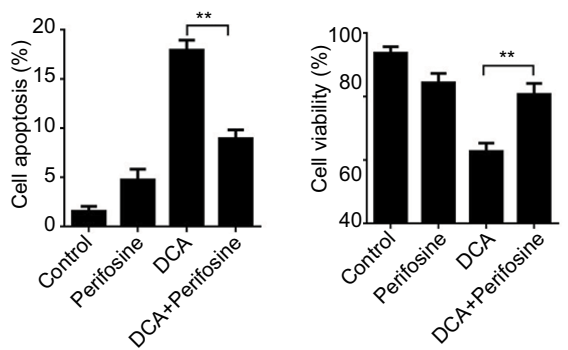
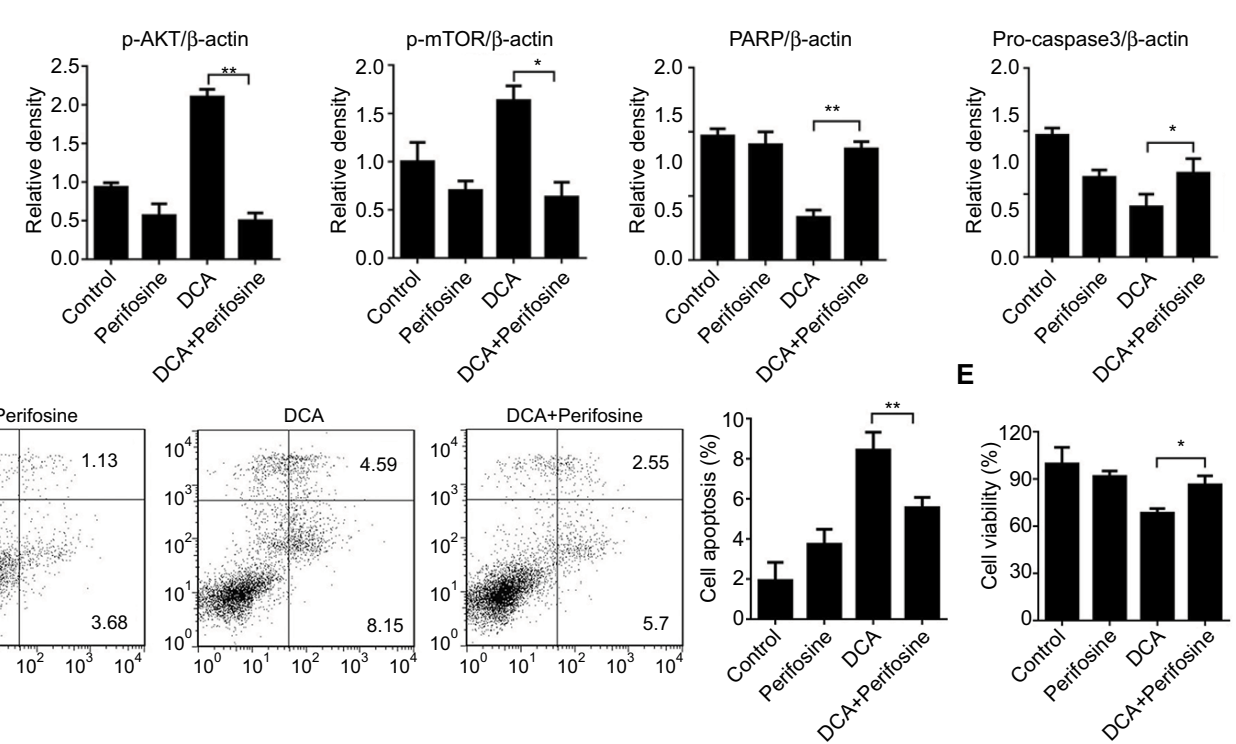

$\mathbf{F}$
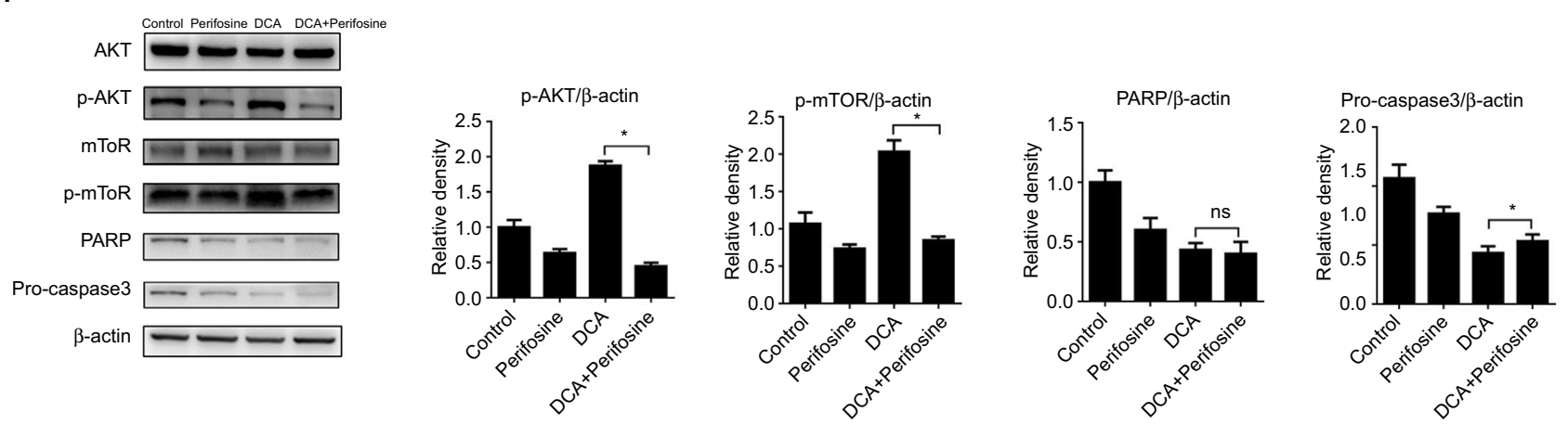

Figure 3 Inhibited AKT pathway downregulates cell death.

Notes: (A) A549 cells and (D) HI975 cells were treated with Perifosine, DCA, or coincubation with DCA plus Perifosine for 24 h. Then, the cell apoptosis was analyzed. The data are presented as the mean \pm SD from three independent experiments $\left({ }^{*} p<0.05\right.$, $\left.{ }^{*} p<0.01\right)$. After treatment with Perifosine, DCA, or coincubation, (B and $\left.\mathbf{E}\right)$ cell viability was determined by MTT assay. The expressions of crucial apoptosis-related proteins and AKT-mTOR pathway-related proteins of (C) A549 cells and (F) HI975 cells were analyzed by Western blot. Comparisons of the intensities were statistically estimated and are presented as the mean \pm SD for three independent experiments $(* p<0.05$, ** $p<0.0 \mathrm{I})$. Abbreviation: DCA, dichloroacetate. 
lation and activation, was used to investigate whether the ATK-mTOR pathway is involved in the autophagy-inhibiting process of DCA. Cells were cotreated with DCA and perifosine for $24 \mathrm{~h}$. Cell apoptosis was almost reduced by half in the coincubation group compared with the DCA treatment group, which is consistent with the cell viability assays (Figure $3 \mathrm{~A}$ and $\mathrm{B}, \mathrm{D}$ and $\mathrm{E}, p<0.05$ ). Then, we examined the levels of crucial apoptosis-related proteins, PARP and procaspase3. AKT phosphorylation was inhibited, and the levels of apoptosis-related proteins were significantly decreased in the cotreatment group (Figure 3C, $p<0.05$ ). These findings were also obtained in the H1975 cells (Figure 3F, $p<0.05$ ).

\section{DCA increases PTX and CDDP sensitivity by reversing the chemotherapy-induced autophagy}

The inhibition of autophagy is considered a new strategy to enhance the sensitivity of chemotherapeutics. ${ }^{23,24}$ To
A
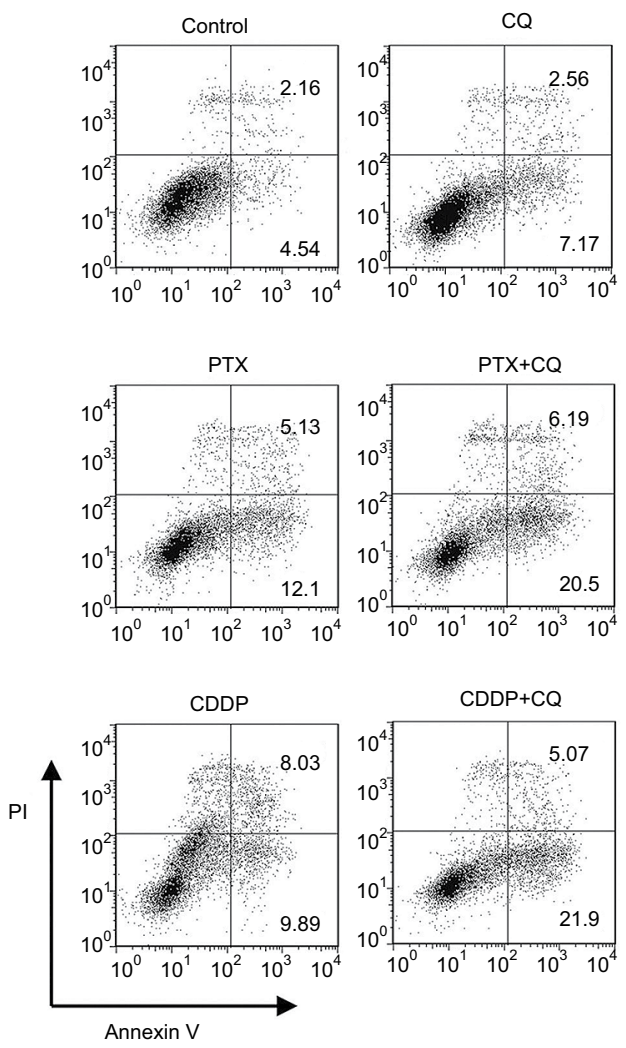

D
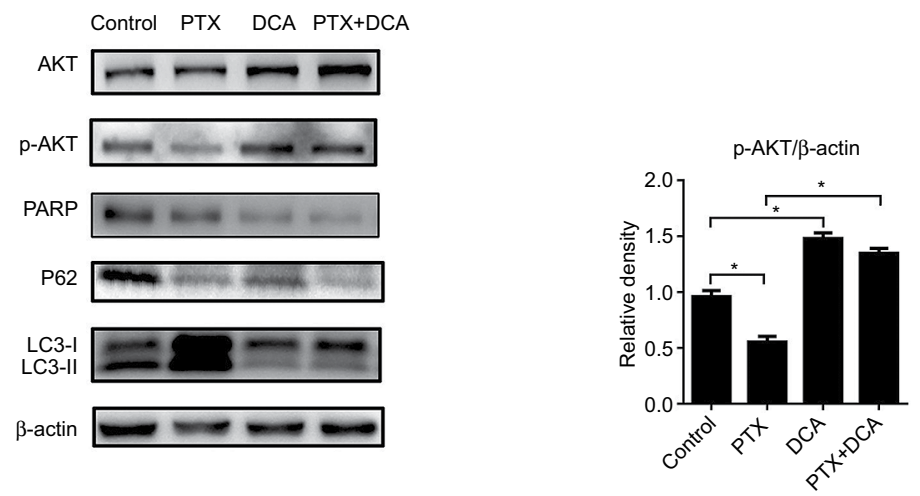
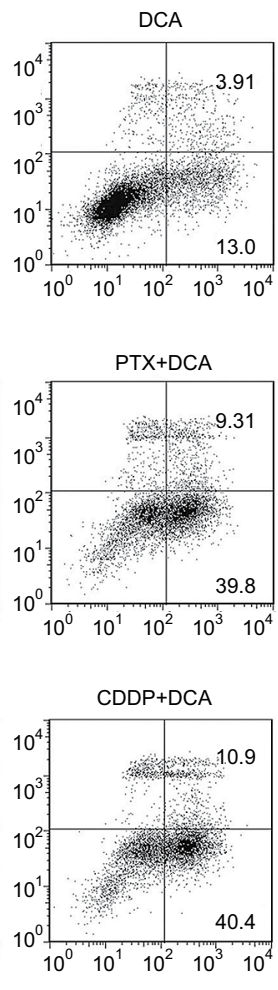

E

B

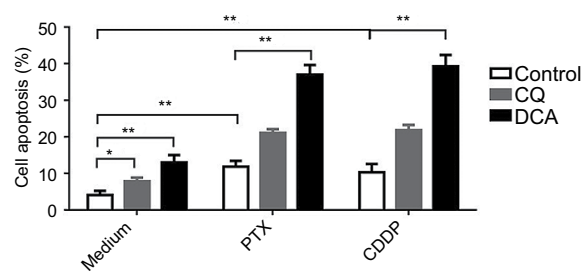

C

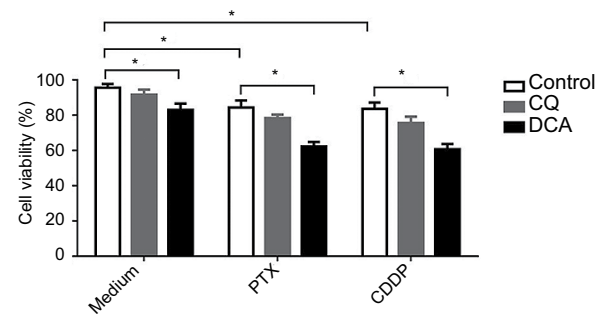

$\mathbf{F}$

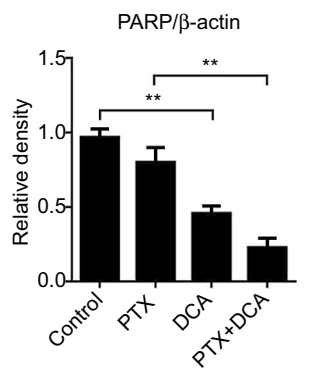

Figure 4 DCA decreased the drug resistance of the tumor cells by inhibiting autophagy.

Notes: Cells were treated with DCA, CQ, chemotherapeutics (PTX or CDDP) or coincubation (DCA or CQ plus chemotherapeutics). Then, the (A and B) cell apoptosis and $(\mathbf{C})$ cell viability assays were analyzed. The data are presented as the mean \pm SD from three independent experiments $(* p<0.05$, $* * p<0.01$ ). (D) Cells were treated with PTX, DCA, or coincubation with DCA plus PTX for $24 \mathrm{~h}$, and then the expressions of crucial apoptosis-related protein, AKT-mTOR pathway-related proteins, and autophagy-related proteins were analyzed by Western blot. (E and F) Comparisons of the intensities of p-AKT and PARP were statistically estimated and are presented as the mean \pm SD for three independent experiments $\left({ }^{*} p<0.05\right.$, $\left.{ }^{*} p<0.0 \mathrm{I}\right)$.

Abbreviations: CDDP, cis-diamminedichloroplatinum; CQ, chloroquine; DCA, dichloroacetate; PTX, paclitaxel. 
investigate whether cotreatment with DCA and a chemotherapeutic agent exerts a synergistic effect to suppress cell growth, A549 cells were cotreated with DCA and PTX or CDDP. As shown in Figure 4A-C, treatment with chemotherapy drugs and DCA increased the levels of apoptosis compared with drug treatment alone or in combination with $\mathrm{CQ}$, and the results were consistent with the cell viability assays $(p<0.05)$. To determine whether DCA can downregulate the autophagy induced by PTX, cells were treated with PTX and DCA for $24 \mathrm{~h}$ and harvested for Western blotting. The levels of autophagy-related proteins were examined. LC3 expression was markedly upregulated by the PTX treatment, and it was significantly decreased when cotreated with DCA. The level of critical apoptosis-related protein, PARP, was significantly decreased after the cotreatment (Figure 4D-F, $p<0.05$ ). All of these results indicate that DCA decreased the drug resistance of A549 cells to PTX by inhibiting autophagy.

\section{DCA potentiated the anticancer efficacy of PTX in vivo by inhibiting autophagy}

Because cotreating cells with PTX and DCA increased the rate of cell death in vitro compared with treatment with either drug alone, we also assessed the in vivo efficacy of the combination therapy of DCA and PTX in a mouse xenograft tumor model. Once the size of the subcutaneous xenograft tumor approached $150 \mathrm{~mm}^{3}$, the mice were separated into four groups: control, DCA, PTX, and DCA plus PTX. The administration of PTX alone did not significantly inhibit tumor growth, but the treatment with PTX plus DCA clearly inhibited the tumor growth and prolonged mouse survival (Figure $5 \mathrm{~A}$, and B, $p<0.05$ ). As shown in Figure $5 \mathrm{C}$, the tumor doubling time of the A549 cells increased significantly from 3 days in the control group to 10 days in the cotreatment group $(p<0.05)$, and the tumor doubling time of the H1975 cells increased from 2.6 to 7.5 days (Figure 5D-F, $p<0.05$ ). These results indicate that DCA can enhance the in vivo anticancer efficacy of PTX.

\section{Treatment with DCA plus PTX significantly inhibited tumor germination}

To investigate whether the combination therapy of DCA and PTX led to the morphologic changes characteristic of apoptosis in the A549 cells in vivo, all tumors were recovered on day 24 , sectioned, stained with TUNEL, and analyzed
A

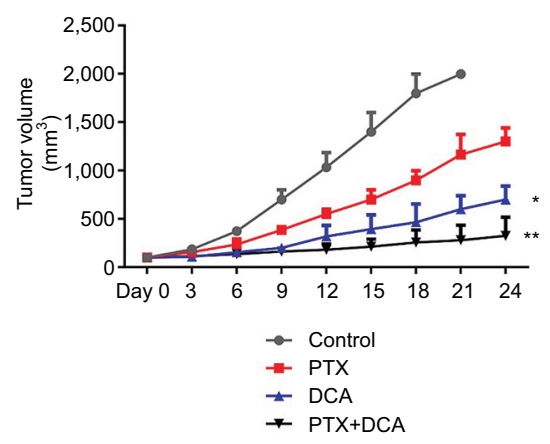

B

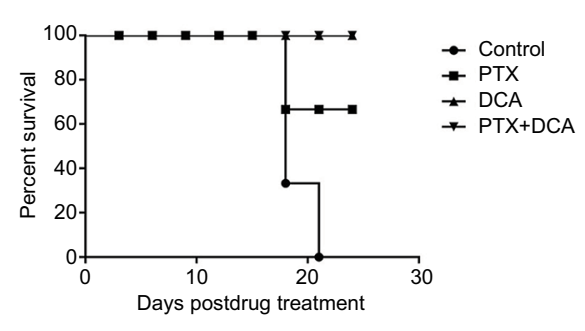

E
C

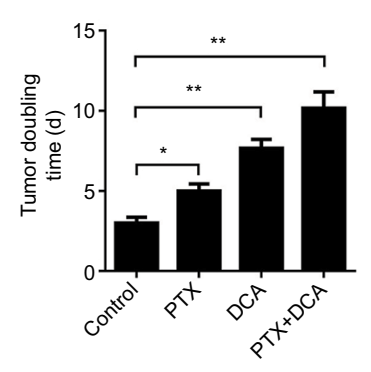

D

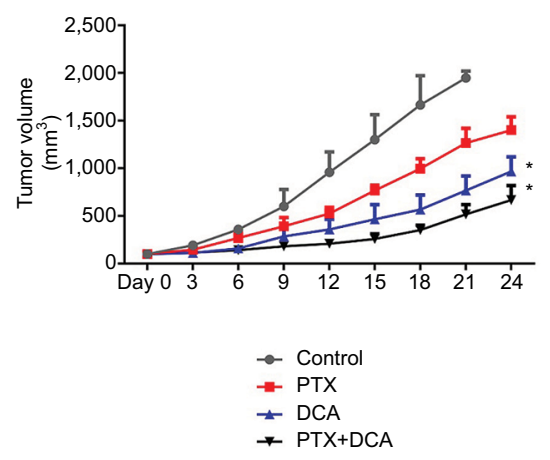

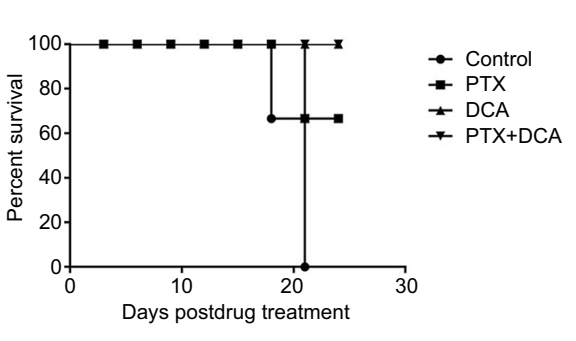

$\mathbf{F}$

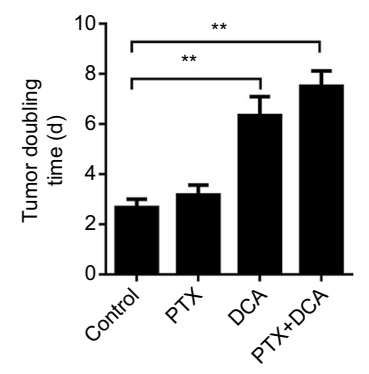

Figure 5 Synergistic inhibition of proliferation in mice treated with PTX and DCA.

Notes: $5 \times 10^{6}$ A549 cells or HI975 cells were implanted into nude mice. When the volume of the subcutaneous xenograft tumor approached 100 mm ${ }^{3}$, the mice were separated into four groups: control, DCA, PTX, and DCA plus PTX. Then, the mice were injected intraperitoneally everyday with DCA (I00 mg/kg/d) plus PTX (20 mg/kg/d) or each alone, using PBS as the control. (A and D) The growth curve of the tumor volumes was monitored every 3 days $\left({ }^{*} p<0.05\right.$, $* * p<0.01$ ). (B and $\left.\mathbf{E}\right)$ Survival curves of mice. Data were analyzed by the log-rank test. (C and F) Tumor doubling time. Data are presented as the mean $\pm S D(* p<0.05, * * p<0.01)$.

Abbreviations: DCA, dichloroacetate; PTX, paclitaxel. 
by immunohistochemistry. There was a minimal increase in the number of TUNEL-positive cells in the DCA or PTX treatment groups relative to the control group. However, the TUNEL-positive cell proportion in the cotreatment group increased dramatically compared with the control or singletreatment groups (Figure 6A).

We then examined the expression of proliferation-related $\mathrm{Ki}-67$ and crucial autophagy protein LC3B in the tumors by immunohistochemical staining. Ki-67 expression in the control mice was significantly higher, whereas its expression was greatly reduced in the tumors of animals receiving the combination treatment, and the expression of LC3B was same as Ki-67. In conclusion, the treatment with DCA plus PTX significantly enhanced apoptosis and reduced Ki-67 and $\mathrm{LC} 3 \mathrm{~B}$ expression in vivo (Figure $6 \mathrm{~A}$ and $\mathrm{B}, p<0.01$ ).

\section{Discussion}

With the development of cancer research, an increasing number of treatment strategies have been applied in lung cancer treatment. ${ }^{25-27}$ Although many lung cancer patients, especially in the late stage or progressive stage, have benefited from EGFR-TKI and PD-1 antibody, it is exorbitantly costprohibitive for most patients. Conventional chemotherapy is still the main approach to treat advanced lung cancer in China. However, multidrug resistance has been a difficult obstacle in cancer treatment; therefore, current research is focus on searching for new ways to enhance chemotherapeutic sensitivity or delay the drug resistance. Recently, autophagy regulation has been considered as a target for anticancer therapy; much evidence has indicated that inhibiting autophagy can promote the tumor-suppressing effect of chemotherapeutics and radiotherapy. ${ }^{23,28-30}$ Usually, most solid carcinomas generate energy through the glycolytic pathway irrespective of whether sufficient oxygen is present; this is called the "Warburg effect". ${ }^{29-31}$ DCA, a small molecule antitumor agent, can reverse this metabolic mode of mitochondria from anaerobic respiration to aerobic respiration, thereby causing tumor cell death. However, whether DCA can be used in combination with other chemotherapeutics is unknown. In this study, we found that cotreatment with DCA and PTX can be more efficacious in suppressing the growth of lung cancer cells in vitro and in vivo. DCA inhibited the protective autophagy employed by cancer cells in response to PTX treatment by activating AKT phosphorylation and promoting cell death. The synergistic effect of these two agents enhances cell death, thereby inhibiting tumor growth more effectively.

As we know, cell metabolism is an effective target for cancer treatment, ${ }^{32}$ and accumulating evidence has demonstrated the antitumor properties of DCA in cell metabolism. However, the effects of DCA are controversial, because different outcomes have been reported in different cancer models. ${ }^{33,34}$ In the present study, we found that the combination of DCA and PTX was more efficacious in inhibiting cell proliferation and enhancing apoptosis than PTX alone. In the subcutaneous tumor xenograft model used in this study, the DCA plus PTX treatment reduced the growth of the A549 cells injected into the mice by $60 \%$. Our data showed that DCA exerted potent anticancer effects and acted synergistically with PTX on A549 cells. Multidrug therapy that acts synergistically may improve the therapeutic efficacy by
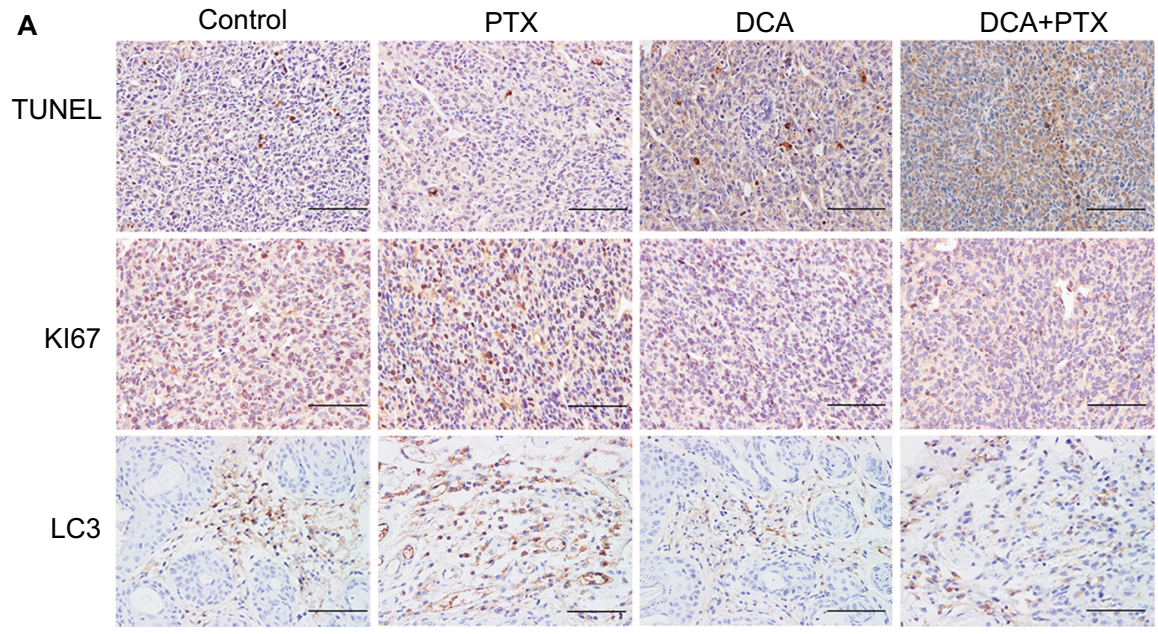

B

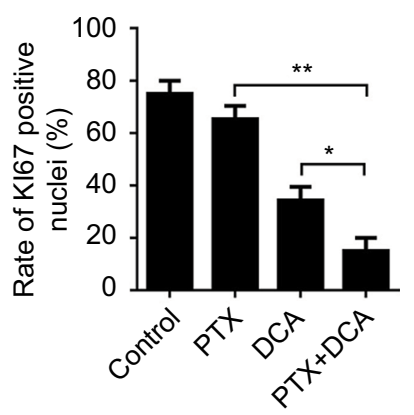

Figure 6 Immunohistochemical examination for tumor apoptosis and proliferation

Notes: (A) On day 24, all tumors were isolated for histopathological examination with TUNEL, Ki-67, and LC3B staining assays (scale bar $=200 \mu \mathrm{m}$ ). (B) The ratio of positive Ki-67 nucleus was analyzed and are presented as the mean \pm SD $(* p<0.05, * * p<0.01)$.

Abbreviations: DCA, dichloroacetate; PTX, paclitaxel. 
decreasing drug toxicity and drug resistance. ${ }^{35}$ In our experiments, DCA reversed the multidrug resistance of A549 cells by inhibiting the autophagy induced by PTX and inducing apoptosis. Therefore, adding DCA to the chemotherapy regimen might decrease the perniciousness of multiple drug resistance in NSCLC.

Autophagy is a highly conserved metabolic pathway in which cells deliver senile or defective proteins, organelles, and other cellular components to lysosomes for degradation to maintain cellular homeostasis. ${ }^{36,37}$ A large body of evidence shows that most chemotherapeutic agents as well as ionizing radiation induce autophagy, leading to multidrug resistance. Adapting autophagy facilitates tumor cell survival in a microenvironment of increased metabolic stress or drug toxicity. Stress-induced autophagy is an important protective strategy that can maintain cell survival, which ultimately results in drug resistance in various types of tumor cells. ${ }^{38}$ In this study, DCA treatment inhibited cellular autophagy and promoted apoptosis. RARA, an autophagy activator, can render the cells more resistant to DCA, indicating that DCA causes cell death by disrupting cancer cell metabolism and inhibiting autophagy. The PI3K-AKT-mTOR pathway is an important negative regulator of autophagy. Therefore, targeting the PI3K-AKT-mTOR signaling can be a novel strategy for cancer therapy. DCA selectively promotes AKT kinase phosphorylation, thereby inhibiting autophagy. Thus, the activation of the PI3K-AKT-mTOR pathway by DCA inhibits PTX-induced autophagy. EGFR-TKIs treatment is reportedly related to the autophagy level of lung cancer cells, and autophagy inhibition might facilitate the antitumor effects of EGFR-TKIs. This might be a novel focal point of lung cancer treatment.

However, some researchers argue that autophagy is a "double-edged sword" for cancer cells, and it can suppress the survival and proliferation in the tumor microenvironment. ${ }^{26,39,40}$ Furthermore, inducing autophagy in cancer was also considered to be a strategy for chemotherapy sensitization. The different functions of autophagy in cancer cells might be caused by the different statuses of cancer cells or tumor types; thus, understanding the underlying functions of autophagy regulation between different cancer cells will shed new light on the designation of strategy against chemotherapy resistance. ${ }^{24}$

Interestingly, DCA appears to have dual characteristics of autophagy regulation in different tumor cells. Lin et $\mathrm{al}^{33}$ have reported that DCA can induce apoptosis in colorectal cancer cells while enhancing autophagy at the same time. It follows that a more complex molecular mechanism is involved in the autophagy regulation of DCA. Exploring the principle of different effects on autophagy of DCA would provide us with new ideas to design new adjuvant chemotherapy drugs through autophagy inhibition.

\section{Conclusion}

Our data showed that autophagy can be activated during chemotherapy, and autophagy inhibition by DCA can facilitate cell death and enhance the tumor cell sensitivity to chemotherapy drugs. Furthermore, autophagy and cancer metabolism were involved in a series of tumor events, such as tumor development, tumor progression, and chemotherapy. Elucidating the relationship between autophagy and metabolism $^{32}$ in different cancer cells might provide us with new opportunities for designing drugs targeting these important cellular events against cancer and drug resistance.

\section{Acknowledgment}

The study was supported by funding from the National Natural Science Foundation of China (81702247) and the Basic Science and Frontier Technology Project of Chongqing for Zheng (cstc2017jcyjAX0048), the National Natural Science Foundation of China for Dai (81472188), and the Clinical Research Project of Xinqiao Hospital for Yu (2015YLC21).

\section{Disclosure}

The authors report no conflicts of interest in this work.

\section{References}

1. Maher AR, Miake-Lye IM, Beroes JM, Shekelle PG. Treatment of Metastatic Non-Small Cell Lung Cancer: A Systematic Review of Comparative Effectiveness and Cost-Effectiveness. Washington (DC): Department of Veterans Affairs (US); 2012.

2. Spiro SG, Porter JC. Lung cancer - where are we today? Current advances in staging and nonsurgical treatment. Am J Respir Crit Care Med. 2002;166:1166-1196.

3. Song W, Tang Z, Li M, et al. Polypeptide-based combination of paclitaxel and cisplatin for enhanced chemotherapy efficacy and reduced side-effects. Acta Biomater. 2014;10:1392-1402.

4. Ward PS, Thompson CB. Metabolic reprogramming: a cancer hallmark even Warburg did not anticipate. Cancer Cell. 2012;21:297-308.

5. Pathak RK, Marrache S, Harn DA, Dhar S. Mito-DCA: a mitochondria targeted molecular scaffold for efficacious delivery of metabolic modulator dichloroacetate. ACS Chem Biol. 2014;9:1178-1187.

6. Kimura T, Takabatake Y, Takahashi A, Isaka Y. Chloroquine in cancer therapy: a double-edged sword of autophagy. Cancer Res. 2013;73: 3-7.

7. Levine B, Kroemer G. Autophagy in the pathogenesis of disease. Cell. 2008;132:27-42.

8. Gomes LR, Vessoni AT, Menck CF. Microenvironment and autophagy cross-talk: Implications in cancer therapy. Pharmacol Res. 2016;107:300-307.

9. Su Z, Yang Z, Xu Y, Chen Y, Yu Q. Apoptosis, autophagy, necroptosis, and cancer metastasis. Mol Cancer. 2015;14:48. 
10. Koren I, Kimchi A. Cell biology. Promoting tumorigenesis by suppressing autophagy. Science. 2012;338:889-890.

11. Rosenfeldt MT, Ryan KM. The role of autophagy in tumour development and cancer therapy. Expert Rev Mol Med. 2009;11:e36.

12. Rubinsztein DC, Codogno P, Levine B. Autophagy modulation as a potential therapeutic target for diverse diseases. Nat Rev Drug Discov. 2012;11:709-730.

13. Egger ME, Huang JS, Yin W, McMasters KM, McNally LR. Inhibition of autophagy with chloroquine is effective in melanoma. $J$ Surg Res. 2013;184:274-281.

14. Rangwala R, Chang YC, Hu J, et al. Combined MTOR and autophagy inhibition: phase I trial of hydroxychloroquine and temsirolimus in patients with advanced solid tumors and melanoma. Autophagy. 2014;10:1391-1402.

15. Vogl DT, Stadtmauer EA, Tan KS, et al. Combined autophagy and proteasome inhibition: a phase 1 trial of hydroxychloroquine and bortezomib in patients with relapsed/refractory myeloma. Autophagy. 2014;10:1380-1390

16. Bonnet S, Archer SL, Allalunis-Turner J, et al. A mitochondria-K+ channel axis is suppressed in cancer and its normalization promotes apoptosis and inhibits cancer growth. Cancer Cell. 2007;11:37-51.

17. Christofk HR, Vander HM, Harris MH, et al. The M2 splice isoform of pyruvate kinase is important for cancer metabolism and tumour growth. Nature. 2008;452:230-233.

18. Tan J, Jiang X, Yin G, et al. Anacardic acid induces cell apoptosis of prostatic cancer through autophagy by ER stress/DAPK3/Akt signaling pathway. Oncol Rep. 2017;38(3):1373-1382.

19. Chen LM, Song TJ, Xiao JH, Huang ZH, Li Y, Lin TY. Tripchlorolide induces autophagy in lung cancer cells by inhibiting the PI3K/AKT/ mTOR pathway and improves cisplatin sensitivity in A549/DDP cells. Oncotarget. 2017;8(38):63911-63922.

20. Wang Z, Wang N, Liu P, Xie X. AMPK and cancer. EXS. 2016;107: 203-226.

21. Umezawa S, Higurashi T, Nakajima A. AMPK: therapeutic target for diabetes and cancer prevention. Curr Pharm Des. 2017;23(25):3629-3644.

22. Gills JJ, Dennis PA. Perifosine: update on a novel Akt inhibitor. Curr Oncol Rep. 2009;11:102-110.

23. Janku F, McConkey DJ, Hong DS, Kurzrock R. Autophagy as a target for anticancer therapy. Nat Rev Clin Oncol. 2011;8:528-539.

24. Rubinsztein DC, Codogno P, Levine B. Autophagy modulation as a potential therapeutic target for diverse diseases. Nat Rev Drug Discov. 2012;11:709-730.
25. Ma W, Gilligan BM, Yuan J, Li T. Current status and perspectives in translational biomarker research for PD-1/PD-L1 immune checkpoint blockade therapy. J Hematol Oncol. 2016;9:47.

26. Dholaria B, Hammond W, Shreders A, Lou Y. Emerging therapeutic agents for lung cancer. $J$ Hematol Oncol. 2016;9:138.

27. Wang S, Cang S, Liu D. Third-generation inhibitors targeting EGFR T790M mutation in advanced non-small cell lung cancer. $J$ Hematol Oncol. 2016;9:34.

28. White E, Karp C, Strohecker AM, Guo Y, Mathew R. Role of autophagy in suppression of inflammation and cancer. Curr Opin Cell Biol. 2010;22:212-217.

29. Mathew R, Karantza-Wadsworth V, White E. Role of autophagy in cancer. Nat Rev Cancer. 2007;7:961-967.

30. Apel A, Herr I, Schwarz H, Rodemann HP, Mayer A. Blocked autophagy sensitizes resistant carcinoma cells to radiation therapy. Cancer Res. 2008;68:1485-1494.

31. Zhou W, Liotta LA, Petricoin EF. The Warburg effect and mass spectrometry-based proteomic analysis. Cancer Genomics Proteomics. 2017;14:211-218.

32. Ho WL, Hsu WM, Huang MC, Kadomatsu K, Nakagawara A. Protein glycosylation in cancers and its potential therapeutic applications in neuroblastoma. J Hematol Oncol. 2016;9:100.

33. Lin G, Hill DK, Andrejeva G, et al. Dichloroacetate induces autophagy in colorectal cancer cells and tumours. Br J Cancer. 2014;111:375-385.

34. Gong F, Peng X, Sang Y, et al. Dichloroacetate induces protective autophagy in LoVo cells: involvement of cathepsin D/thioredoxin-like protein 1 and Akt-mTOR-mediated signaling. Cell Death Dis. 2013;4:e913.

35. Chou TC. Theoretical basis, experimental design, and computerized simulation of synergism and antagonism in drug combination studies. Pharmacol Rev. 2006;58:621-681.

36. Eskelinen EL. The dual role of autophagy in cancer. Curr Opin Pharmacol. 2011;11:294-300.

37. Ravikumar B, Sarkar S, Davies JE, et al. Regulation of mammalian autophagy in physiology and pathophysiology. Physiol Rev 2010;90:1383-1435.

38. Naveed S, Aslam M, Ahmad A. Starvation based differential chemotherapy: a novel approach for cancer treatment. Oman Med J. 2014;29:391-398.

39. Yoshida GJ. Therapeutic strategies of drug repositioning targeting autophagy to induce cancer cell death: from pathophysiology to treatment. J Hematol Oncol. 2017;10:67.

40. Choi AM, Ryter SW, Levine B. Autophagy in human health and disease. N Engl J Med. 2013;368:651-662.
Cancer Management and Research

\section{Publish your work in this journal}

Cancer Management and Research is an international, peer-reviewed open access journal focusing on cancer research and the optimal use of preventative and integrated treatment interventions to achieve improved outcomes, enhanced survival and quality of life for the cancer patient. The manuscript management system is completely online and includes

\section{Dovepress}

a very quick and fair peer-review system, which is all easy to use. Visit $\mathrm{http}: / / \mathrm{www}$.dovepress.com/testimonials.php to read real quotes from published authors. 